\title{
Multiple Andreev reflections in hybrid multiterminal junctions
}

\author{
M. Houzet ${ }^{1}$ and P. Samuelsson ${ }^{2}$ \\ ${ }^{1}$ SPSMS, UMR-E 9001, CEA-INAC/UJF-Grenoble 1, F-38054 Grenoble, France \\ ${ }^{2}$ Division of Mathematical Physics, Lund University, Box 118, S-221 00 Lund, Sweden
}

(Dated: October 30, 2018)

\begin{abstract}
We investigate theoretically charge transport in hybrid multiterminal junctions with superconducting leads kept at different voltages. It is found that multiple Andreev reflections involving several superconducting leads give rise to rich subharmonic gap structures in the current-voltage characteristics. The structures are evidenced numerically in junctions in the incoherent regime.
\end{abstract}

PACS numbers: 72.10.-d, 73.23.-b, 74.25.fc, 74.45.+c

The mechanism of charge transfer across an interface between a normal $(\mathrm{N})$ conductor and a superconductor (S) at subgap energies is Andreev reflection (AR) [1]. In an $\mathrm{AR}$ an electron incident from $\mathrm{N}$ is retroreflected as a hole, creating at the same time a Cooper pair in S. In SNS-junctions subjected to a voltage bias $V$, particles can undergo several ARs at the NS-interfaces, gaining energy $e V$ at each traversal of the junction, and escape out in the leads at energies above the superconducting gap $\Delta$. This process of multiple Andreev reflections [2, 3] (MAR) is responsible for the charge transport at voltages $\mathrm{eV}<2 \Delta$ and gives rise to a subharmonic gap structure (SGS) at $e V=2 \Delta / n$ ( $n$ integer) in the current-voltage characteristics.

Almost thirty years after its theoretical description, MAR still attracts a lot of interest. In recent years, the strongly non-equilibrium electron distribution caused by MAR was measured in metallic diffusive junctions [4]. An enhanced shot noise, due to the multiparticle character of MAR-transport, was experimentally demonstrated in tunnel [5], metallic [6], and atomic point contact [7] junctions. Superconducting junctions based on new materials and nanoscale systems such as carbon nanotubes [8], semiconductor nanowires [9], and graphene flakes [10] has allowed for an investigation of the interplay of MAR and resonant transport, charging effects and Kondo physics, as well as MAR-transport of Dirac electrons.

While most experimental and theoretical investigations of MAR have concerned two-terminal structures, multiterminal geometries with all superconducting leads have also been studied. Phase dependent MAR-transport in SNS-interferometers was investigated experimentally in a diffusive conductor [11] and theoretically in a single mode junction [12]. In an incoherent three-terminal junction, the current cross-correlation between $\mathrm{S}$ leads was predicted to be strongly enhanced due to MARs [13].

In all these works however, only one bias voltage was applied between the different leads [14]. In this article, we show that a much richer picture for SGS and MAR-transport manifests in multiterminal structures with arbitrary bias voltages. Specifically, we address a three-superconducting-terminal structure and predict

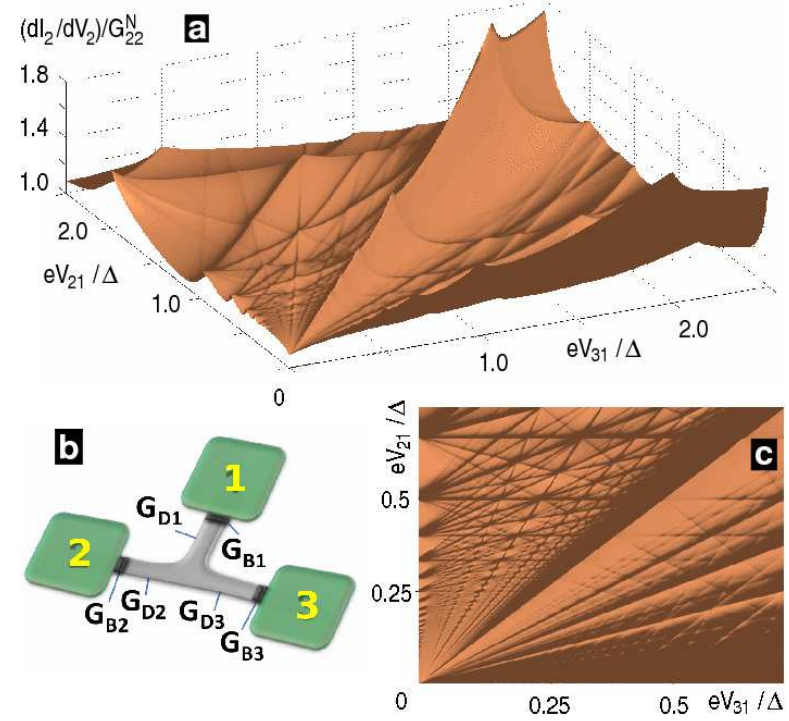

FIG. 1: (a) The differential conductance $G_{22}=\partial I_{2} / \partial V_{2}$ normalized with its normal state value as a function of bias voltages $V_{21}$ and $V_{31}$ in a symmetric three-superconductingterminal junction in the incoherent regime. The conductances $G_{B \alpha}=\left(2 e^{2} / h\right) N \Gamma$ with $\Gamma=0.7$ and negligible resistances $1 / G_{D \alpha}$ of the diffusive regions. The temperature $k T=0.01 \Delta$. (b) Geometry of the junction with superconducting terminals $\alpha=1,2,3$, barrier conductances $G_{B \alpha}$ and conductances $G_{D \alpha}$, of the diffusive regions shown. (c) Top view of low voltage region of (a).

SGS when (see Fig. 1)

$$
\begin{aligned}
& p V_{21}+q V_{31}=2 \Delta / e, \quad \text { or } \\
& p V_{21}+q V_{31}=0 .
\end{aligned}
$$

Here, $p$ and $q$ are integers and $V_{21}=V_{2}-V_{1}$ (resp. $V_{31}=$ $V_{3}-V_{1}$ ) is the bias voltage between leads 2 and 1 (resp. 3 and 1 ). This prediction is quite general and could thus be tested in a broad range of hybrid systems.

In the following, we first provide a physically intuitive description of the new SGS features in multiterminal junctions with all superconducting leads. Thereafter, we present a detailed investigation of the SGS and MARtransport for junctions in the incoherent regime. This is 


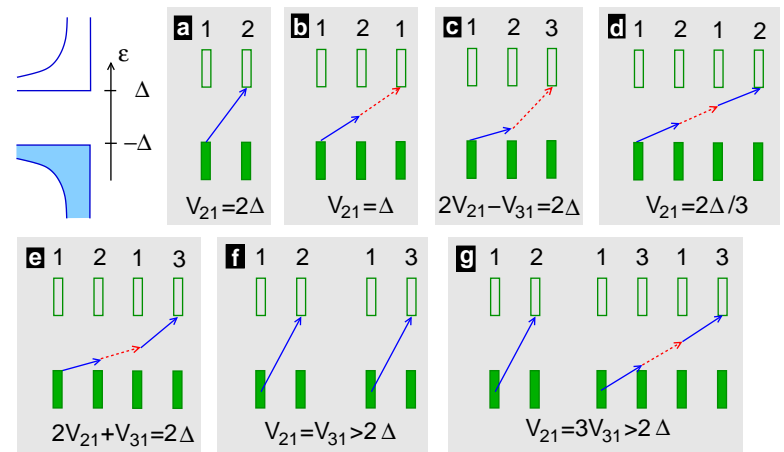

FIG. 2: Examples of elementary processes determining the SGS lines (see text). The filled (empty) rectangles represent filled (empty) states in the gapped energy spectrum of the leads (upper left fig.). Full (dashed) arrows represent an electron (hole) propagating between two leads. The slope of each arrow is set by the bias voltages. An AR takes place at the intersection of full and dashed arrows.

then contrasted with the result for multiterminal structures having both normal and superconducting leads.

Let us first discuss the SGS given by Eq. (1a). As in standard two-terminal junctions, the SGS occur at bias voltages for which new transport processes in energy space become possible, taking particles from below to above the superconducting gap. In the absence of AR, direct transmission of an electron through the structure takes place at $\left|V_{\alpha \beta}\right|>2 \Delta / e\left(V_{\alpha \beta}=V_{\alpha}-V_{\beta}\right)$; the process is illustrated in Fig. 2 2 . This determines the SGS lines: $V_{21}= \pm 2 \Delta / e, V_{31}= \pm 2 \Delta / e$, and $V_{21}-V_{31}= \pm 2 \Delta / e$. Formally, these can be interpreted as the usual MAR features with $n=1$.

Consider then a process where an electron injected from lead $\alpha$ is Andreev reflected into a hole at lead $\beta$ and finally escapes in lead $\gamma$. The threshold voltage for this process is $V_{\beta \alpha}-V_{\gamma \beta}=2 \Delta / e$. When $\gamma=\alpha$, we recognize the usual MAR features for leads $\alpha$ and $\beta$ with $n=2$, see Fig. 2 $\mathrm{b}$. When $\gamma \neq \alpha$, it determines a new set of SGS lines at $V_{21}+V_{31}= \pm 2 \Delta / e, 2 V_{21}-V_{31}= \pm 2 \Delta / e$, and $V_{21}-2 V_{31}= \pm 2 \Delta / e$ (see Fig. 22).

Similarly, the processes where an incoming electron undergoes two ARs before escaping out at the upper gap edge in a S-lead determine the standard set of MAR features with $n=3$, see Fig. $2 \mathrm{~d}$, as well as the lines: $V_{21}+2 V_{31}= \pm 2 \Delta / e, V_{21}-3 V_{31}= \pm 2 \Delta / e$, and $2 V_{21}-3 V_{31}= \pm 2 \Delta / e$, plus three other lines obtained by interchanging the indices 2 and 3 (see Fig. 2e). The generalization to arbitrary numbers of ARs is straightforward and yields the lines (1a).

Now, we discuss the SGS lines given by Eq. (1b). These lines, in contrast to those in Eq. (1a), arise due to the interplay of two different processes that take a quasiparticle from below the gap in lead $\alpha$ to the upper gap edge in lead $\beta$ or $\gamma$. In the absence of $A R$, for e.g. $V_{21}=V_{31}>2 \Delta / e$, an electron injected at en- ergy $\varepsilon<-\Delta-e V_{1}$ in lead 1 can be directly transmitted through the structure, out into lead 2 or 3 (see Fig. 25). However, for $V_{21}>V_{31}\left(V_{31}>V_{21}\right)$ there is an energy interval $\Delta-e V_{2}<\varepsilon<\Delta-e V_{3}\left(\Delta-e V_{3}<\varepsilon<\Delta-e V_{2}\right)$ for which injected electrons only can escape out into lead 2 (3). This opening up of one and closing of another transport process is the origin of the SGS-line at $V_{21}=V_{31}$, with $\left|V_{21}\right|>2 \Delta / e$. Similarly, we have SGSlines at $V_{31}=0$ with $\left|V_{21}\right|>2 \Delta / e$, and $V_{21}=0$ with $\left|V_{31}\right|>2 \Delta / e$, due to the interplay between processes with no AR.

For one $\mathrm{AR}$ in one of the processes we find in the same way SGS-lines at $V_{21}=2 V_{31}$ with $\left|V_{21}\right|>2 \Delta / e$, $V_{31}=2 V_{21}$ with $\left|V_{31}\right|>2 \Delta / e$ and $V_{21}=-V_{31}$ with $\left|V_{21}\right|>\Delta / e$. If in total two ARs in the two processes are considered we find first the same SGS-lines as for zero AR but with a voltage threshold at $\Delta$. Second, we also have the line at $2 V_{31}=3 V_{21}$ with $\left|V_{21}\right|>\Delta / e$ (see Fig. $2 \mathrm{~b}$ ) plus five more lines obtained by permutation of the indices 1, 2 and 3. Again, the generalization to arbitrary numbers of ARs yields the lines in Eq. (1b).

On the whole, the SGS lines are determined by Eq. (1) and they only get smeared at temperatures $k T \sim \Delta$, close to the superconducting critical temperature of the leads. We also expect that a good contact between the S-leads and the intermediate N-region is required to observe many lines. For poor interfaces, AR is strongly suppressed and only the lines corresponding to a small number of ARs are visible. We stress that Eq. (10) is quite generic; it holds for any incoherent as well as short, coherent junction. For long, coherent junctions, i.e. with normal-state dwell time comparable to $\hbar / \Delta$, additional SGS features related to the inverse dwell time (Thouless energy) are expected [15, 16]. The appearance of the SGS-features - steps, peaks/dips or cusps in the voltage dependence of the differential conductances or secondorder derivatives - depends on the transparencies of the NS-interfaces and on the model for electron propagation in the $\mathrm{N}$ region [17].

In the following, we investigate the SGS-lines and the MAR-transport in a three-terminal normal diffusive metal contacted to the $\mathrm{S}$ leads through barriers with arbitrary transparencies (see Fig. 1b). We restrict ourselves to the incoherent case: The length of the normal arms is assumed to be much larger than a pair-breaking length set either by magnetic impurities or by an external magnetic field. Then, the coherence between an incident electron and Andreev reflected hole does not extend into the normal metal and no non-dissipative Josephson current can flow through the structure. In particular, the electronic properties of the normal metal are fully described by the energy-resolved nonequilibrium distribution functions for electrons and holes. 
The current flowing through each arm,

$$
I_{\alpha}=\frac{e}{h} \int d \varepsilon\left[i_{\alpha}^{e}(\varepsilon)-i_{\alpha}^{h}(\varepsilon)\right],
$$

is decomposed into spectral currents for electrons,

$$
\begin{aligned}
i_{\alpha}^{e}(\varepsilon)= & \mathcal{T}_{\alpha}\left(\varepsilon+e V_{\alpha}\right)\left[f^{e}(\varepsilon)-f_{0}\left(\varepsilon+e V_{\alpha}\right)\right] \\
& +\mathcal{A}_{\alpha}\left(\varepsilon+e V_{\alpha}\right)\left[f^{e}(\varepsilon)-f^{h}\left(\varepsilon+2 e V_{\alpha}\right)\right],
\end{aligned}
$$

and holes, $i_{\alpha}^{h}(\varepsilon)=-i_{\alpha}^{e}(-\varepsilon)$. Current conservation at the connection point of the three arms, called the central node, imposes

$$
\sum_{\alpha} i_{\alpha}^{e}(\varepsilon)=0, \quad \sum_{\alpha} i_{\alpha}^{h}(\varepsilon)=0 .
$$

Here, $f^{e}(\varepsilon)$ and $f^{h}(\varepsilon)=1-f^{e}(-\varepsilon)$ are the distribution functions for electrons and holes in the central node, $f_{0}$ is the Fermi distribution function at temperature $T$, and $\mathcal{T}_{\alpha}$ and $\mathcal{A}_{\alpha}$ are the coefficients for normal transmission and AR between the lead $\alpha$ and the node. We further assume that the barriers between the leads and the arms are point contacts characterized by a number of channels $N_{\alpha}$ and a transparency $\Gamma_{\alpha}$ in the normal state, with conductance $G_{B \alpha}=\left(2 e^{2} / h\right) N_{\alpha} \Gamma_{\alpha}$. Then,

$$
\begin{aligned}
\mathcal{T}_{\alpha}(\varepsilon) & =\frac{N_{\alpha} T_{\alpha}(\varepsilon) g_{D \alpha}}{g_{D \alpha}+N_{\alpha} T_{\alpha}(\varepsilon)}, \\
\mathcal{A}_{\alpha}(\varepsilon) & =\frac{N_{\alpha} A_{\alpha}(\varepsilon) g_{D_{\alpha}}^{2}}{\left[g_{D \alpha}+N_{\alpha} T_{\alpha}(\varepsilon)\right]\left[g_{D \alpha}+N_{\alpha}\left\{T_{\alpha}(\varepsilon)+2 A_{\alpha}(\varepsilon)\right\}\right]} .
\end{aligned}
$$

Here, $G_{D \alpha}=\left(2 e^{2} / h\right) g_{D \alpha}$ are the conductances of the normal arms and $T_{\alpha}(\varepsilon)$ and $A_{\alpha}(\varepsilon)$ are the normaltransmission and AR probabilities at a single NSinterface [18]. Eq. (5) allows to describe the crossover between two limiting cases: When the diffusive wire is in good contact with the leads, $G_{D \alpha} \ll G_{B \alpha}$, they reduce to $\mathcal{T}_{\alpha}(\varepsilon)=g_{D \alpha} \theta(|\varepsilon|-\Delta)$ and $\mathcal{A}_{\alpha}(\varepsilon)=\left[g_{D \alpha} / 2\right] \theta(\Delta-|\varepsilon|)$ [19]. On the other hand, when $G_{D \alpha} \gg G_{B \alpha}$, they describe an incoherent chaotic or diffusive dot attached to the leads through point contacts 15].

In order to calculate the currents $I_{\alpha}$ in (2) at given bias voltages, we need the distribution functions $f^{e}(\varepsilon)$ and $f^{h}(\varepsilon)$ in the central node. They are determined by an infinite system of linear equations (3)-(4) relating them at various energies. In general, we solve this system numerically with a standard Jacobi algorithm after projection on a discrete energy grid. The convergence is very fast (only a few iterations are necessary), yielding strongly nonequilibrium distribution functions with sharp peaks and dips at energies in and around the gapped region. The differential conductances $G_{\alpha \beta}=\partial I_{\alpha} / \partial V_{\beta}$ are then obtained by numerical differentiation.

We illustrate the results for a chaotic dot symmetrically contacted to the leads. The SGS lines (10) in the differential conductance are clearly visible in Fig. 1a,c. For clarity, we display only the result for positive values $V_{21}, V_{31} \geq 0$. The SGS lines appear in any local and nonlocal conductance and are robust for temperatures up to the critical temperature of the leads (not shown).

In addition to the SGS-lines we investigate the excess current and the conductance at low bias. Standard methods for MAR-transport at large [18] or small voltage 20] in two-terminal junctions are readily generalized to the multiterminal geometry considered here. We provide the results in the following. At large bias voltages $\left|V_{21}\right|,\left|V_{31}\right|,\left|V_{32}\right| \gg \Delta / e$ the currents flowing through the leads are close to their normal state value:

$$
I_{\alpha}^{N}=\sum_{\beta \neq \alpha} \frac{G_{\alpha} G_{\beta}}{G_{\Sigma}}\left(V_{\alpha}-V_{\beta}\right),
$$

where $G_{\alpha}^{-1}=G_{B \alpha}^{-1}+G_{D \alpha}^{-1}$ and $G_{\Sigma}=\sum_{\alpha} G_{\alpha}$. The effect of the $\mathrm{S}$ leads is to induce an excess current that takes a finite value at large voltages, $I_{\alpha}=I_{\alpha}^{N}+I_{\alpha}^{\text {exc }}$, where for $k T \ll \Delta$

$$
I_{\alpha}^{\mathrm{exc}}=\frac{\Delta}{2 e} \sum_{\beta, \gamma, \eta}\left(\delta_{\beta \alpha}-\delta_{\gamma \alpha}\right) \frac{G_{\beta} \Lambda_{\gamma} G_{\eta}}{G_{\Sigma}} \operatorname{sign}\left(V_{\eta \gamma}\right),
$$

and

$$
\Lambda_{\alpha}=\int \frac{d E}{\Delta} \frac{\mathcal{T}_{\alpha}(E)+2 \mathcal{A}_{\alpha}(E)-h G_{\alpha} /\left(2 e^{2}\right)}{\mathcal{T}_{\alpha}(E)+2 \mathcal{A}_{\alpha}(E)+h\left(G_{\Sigma}-G_{\alpha}\right) /\left(2 e^{2}\right)} .
$$

When the resistance of the interfaces is negligible $\left(G_{D \alpha} \ll G_{B \alpha}\right)$, we obtain $\Lambda_{\alpha}=0$ and thus $I_{\alpha}^{\text {exc }}=0$. However, in the general case the excess current remains finite. In the case of a symmetric device with $G_{\alpha} \equiv G$ and $\Lambda_{\alpha} \equiv \Lambda$ all identical, the excess current (7) reduces to $I_{\alpha}^{\text {exc }}=I_{0} \sum_{\beta} \operatorname{sign}\left(V_{\alpha}-V_{\beta}\right)$ where $I_{0}=G \Lambda \Delta /(2 e)$. Thus it can only take one of the values $I_{\alpha}^{\text {exc }}=0, \pm 2 I_{0}$, depending on the bias voltages.

At energies inside the gap, $|\varepsilon|<\Delta$, the coefficient for normal transmission vanishes, $\mathcal{T}_{\alpha}=0$. Equations (3)-(4) at vanishing bias then reduce to a diffusion equation in energy space:

$$
\frac{\partial}{\partial \varepsilon}\left(\mathcal{D}(\varepsilon) \frac{\partial f^{e}(\varepsilon)}{\partial \varepsilon}\right)=0
$$

where $\mathcal{D}(\varepsilon)=\sum_{\alpha \beta}\left(V_{\alpha}-V_{\beta}\right)^{2} \mathcal{A}_{\alpha}(\varepsilon) \mathcal{A}_{\beta}(\varepsilon) / \sum_{\alpha} \mathcal{A}_{\alpha}(\varepsilon)$. Outside the gap, $|\varepsilon|>\Delta$, $f^{e}(\varepsilon)=f_{0}(\varepsilon)$, giving boundary conditions $f^{e}(-\Delta)=1$ and $f^{e}(\Delta)=0$ at $k T \ll \Delta$. Eq. (9) is then readily solved and we find the current through lead $\alpha$ :

$$
I_{\alpha}=\frac{e^{2}}{h}\left(\int_{-\Delta}^{\Delta} \frac{d \varepsilon}{\mathcal{D}(\varepsilon)} \frac{\partial \mathcal{D}(\varepsilon)}{\partial V_{\alpha}}\right) / \int_{-\Delta}^{\Delta} \frac{d \varepsilon}{\mathcal{D}(\varepsilon)}
$$

For a diffusive region contacted to the leads through perfectly transparent interfaces (all $\Gamma_{\alpha}=1$ ), the coefficients 
$\mathcal{A}_{\alpha}$ are constant inside the gap [18] and one finds:

$$
I_{\alpha}^{\text {low } V}=\sum_{\beta \neq \alpha} \frac{\tilde{G}_{\alpha} \tilde{G}_{\beta}}{\tilde{G}_{\Sigma}}\left(V_{\alpha}-V_{\beta}\right),
$$

where $\tilde{G}_{\alpha}^{-1}=\left(2 G_{B \alpha}\right)^{-1}+G_{D \alpha}^{-1}$ and $\tilde{G}_{\Sigma}=\sum_{\alpha} \tilde{G}_{\alpha}$. Eq. (11) reduces to the normal state result (6) at $G_{B \alpha} \gg$ $G_{D \alpha}$, while it predicts local and nonlocal conductance doubling compared to the normal state result at $G_{B \alpha} \ll$ $G_{D \alpha}$. For a symmetric structure with identical arms (arbitrary transparencies), we again obtain an expression similar to (11) but with (all identical) effective conductances: $\tilde{G}_{\alpha}=\left(4 e^{2} / h\right) / \int_{-\Delta}^{\Delta} d \varepsilon /\left[2 \Delta \mathcal{A}_{\alpha}(\varepsilon)\right]$.

From these analyses we find that for a diffusive structure with negligible interface resistances, the currents flowing through it at $k T \ll \Delta$ coincide with their normal state values both in the low and high voltage regions (no excess current). Numerical calculations for intermediate voltages and temperatures up to $k T \sim \Delta$ also show no SGS line. Therefore, the MARs neither show up in the local [20] nor in the nonlocal conductances.

Let us now compare the results with those for hybrid multiterminal junctions with two $\mathrm{S}$ leads (bias voltages $V_{2}$ and $V_{3}$ ) and one $\mathrm{N}$ lead (bias voltage $V_{1}$ ). There is again a complex pattern of SGS lines at voltages:

$$
\begin{aligned}
& n\left(V_{21}-V_{31}\right)=2 \Delta / e \quad(n \text { integer }), \\
& n V_{21}-(n-1) V_{31}= \pm \Delta / e, \\
& V_{21}=V_{31} \quad \text { at } \quad\left|V_{21}\right|>\Delta / e .
\end{aligned}
$$

The lines (12a) are related to standard MARs between the S leads, surviving up to the superconducting critical temperature. In contrast, the lines (12b) arise when an electron is injected from the $\mathrm{N}$ lead, at the chemical potential, and is emitted at the gap edge of one of the $\mathrm{S}$ leads after several ARs between them. Since these lines are dependent on the position of the chemical potential in the $\mathrm{N}$ lead, they are smeared already at finite temperatures $k T \ll \Delta$. The last line (12c) arises due to the interplay of two processes where a particle emitted from below the chemical potential in the $\mathrm{N}$ lead reaches the upper gap edge in one of the two S-leads. At zero temperature, this process has a voltage threshold at $\left|V_{21}\right|>\Delta / e$. At finite temperature $k T \ll \Delta$, the threshold is shifted toward lower voltage $\left|V_{21}\right| \gtrsim(\Delta-k T) / e$ due to the smearing of the distribution function near the chemical potential in the $\mathrm{N}$ lead. It eventually shades away at $k T \sim \Delta$.

The set of equations (3)-(44) can also be used to determine the currents in an incoherent multiterminal junction with $\mathrm{N}$ and $\mathrm{S}$ leads ( $\Delta \rightarrow 0$ in $\mathrm{N}$ leads). The lines (12) in the differential conductances and their temperature dependence are clearly visible in Fig. 3 ,

Thus we have formulated a theory for charge transport in the incoherent three-terminal structure shown in Fig. 1. It could be easily generalized to a circuit theory
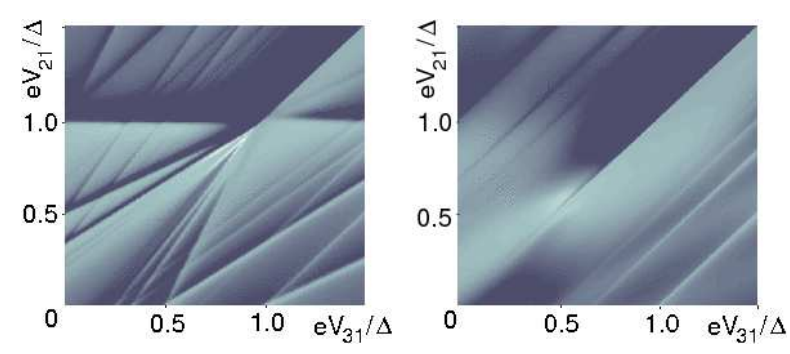

FIG. 3: Top view (similar to Fig. 1) of the differential conductance $G_{21}$ for lead 1 normal and 2,3 superconducting. The conductances $G_{B \alpha}$ and $G_{D \alpha}$ are the same as in Fig. 1 and temperatures are (a) $k T=0.01 \Delta$ and (b) $k T=0.15 \Delta$.

for an arbitrary number of leads, incoherent nodes and connectors between them. Other connectors than the point contact and incoherent diffusive wire in series considered here could also be addressed. For instance, the quantum coherence of the AR process in vicinity of the $S$ leads would lead to renormalized values of the coefficients $T_{\alpha}(\varepsilon)$ and $A_{\alpha}(\varepsilon)$, see Ref. 21]. Our approach could also be extended to calculations of the current correlations 13] and the full counting statistics [22].

In conclusion, we have addressed nonlocal currents in hybrid multiterminal junctions. We have shown that multiple Andreev reflections involving several leads give rise to a much richer subharmonic gap structure than previously investigated in two-terminal junctions. An experimental test of our predictions is feasible in presently available superconducting systems.

We thank Yu. Nazarov for discussion. MH acknowledges support from ANR-07-NANO011 ELEC-EPR and PS support from the Swedish VR.

[1] A.F. Andreev, Sov. Phys. JETP 19, 1228 (1964).

[2] T. M. Klapwijk, G. E. Blonder, and M. Tinkham, Physica B C 109-110, 1657 (1982).

[3] M. Octavio et al., Phys. Rev. B 27, 6739 (1983).

[4] F. Pierre et al., Phys. Rev. Lett. 86, 1078 (2001).

[5] P. Dieleman et al., Phys. Rev. Lett. 79, 3486 (1997).

[6] X. Jehl et al., Phys. Rev. Lett. 83, 1660 (1999); T. Hoss et al., Phys. Rev. B 62, 4079 (2000); C. Hoffmann et al., Phys. Rev. B 70, 180503 (2004).

[7] R. Cron et al., Phys. Rev. Lett. 86, 4104 (2001).

[8] M.R. Buitelaar et al., Phys. Rev. Lett. 91, 057005 (2003); P. Jarillo-Herrero, J. A. van Dam, and L. Kouwenhoven, Nature 439, 953 (2006); H.I. Jorgensen et al., Phys. Rev. Lett. 96, 207003 (2006).

[9] Y.J. Doh et al, Science 309, 272 (2005); Jie Xiang et al. Nature Nanotechnology 1, 208 (2006).

[10] H.B. Heersche et al., Nature 446, 56 (2007).

[11] J. Kutchinsky et al., Phys. Rev. B 56, R2932 (1997).

[12] J. Lantz et al., Phys. Rev. B 65, 134523 (2002).

[13] S. Duhot et al., Phys. Rev. Lett. 102, 086804 (2009).

[14] We note that in Ref. [4] a superconducting probe was 
tunnel coupled to a diffusive SNS-junction, giving only a few of the SGS-lines in our Eq. (1).

[15] P. Samuelsson et al., Phys. Rev. B 65, 180514 (2002).

[16] J. C. Cuevas et al., Phys. Rev. B 73, 184505 (2006).

[17] For a recent discussion see e.g. Quantum transport, Yu. V. Nazarov and Ya. M. Blanter, Cambridge University Press (2009).

[18] G. E. Blonder et al., Phys. Rev. B 25, 4515 (1982).
[19] K. E. Nagaev and M. Büttiker, Phys. Rev. B 63, 081301 (2001).

[20] K. E. Nagaev, Phys. Rev. Lett. 86, 3112 (2001).

[21] E. V. Bezuglyi et al., Phys. Rev. B 62, 14439 (2000).

[22] S. Pilgram and P. Samuelsson, Phys. Rev. Lett. 94, 086806 (2005). 